\title{
Parietal Lobe and Occipital Lobe Ependymal Tumor
}

National Cancer Institute

\section{Source}

National Cancer Institute. Parietal Lobe and Occipital Lobe Ependymal Tumor. NCI

Thesaurus. Code C131581.

An ependymal tumor affecting the parietal and occipital lobes of the brain. 